DOI 10. 18307/2018. 0607

(C) 2018 by Journal of Lake Sciences

\title{
水动力条件对沉积物一水界面氧通量的影响”
}

\author{
郑阳华 ${ }^{1}$, 邹浩东 ${ }^{2}$, 何 强 $^{2}$, 李 宏 $^{2}$, 张 青 $^{3}$, 陈德敏 ${ }^{4}$, 艾海男 ${ }^{2 * *}$ \\ ( 1 : 重庆市环境保护局, 重庆 401147) \\ ( 2 : 重庆大学三峡库区生态环境教育部重点实验室, 重庆 400044) \\ (3: 长沙市规划设计院有限责任公司, 长沙 410000) \\ (4:重庆大学法学院, 重庆 400044)
}

\begin{abstract}
摘 要: 氧环境决定了水体沉积物中各种生命所需元素的最终归趋, 沉积物一水界面是水相与沉积物相氧传递的重要场 所, 而水动力条件是影响沉积物一水界面氧传递的重要因素. 选择三峡库区一级支流御临河为研究对象, 根据长年监测 数据建立实验室模型, 采用声学多普勒流速测试仪及微电极测试系统构建了非侵人式浴度相关测试系统, 探究了不同 水动力条件对沉积物一水界面氧通量的影响. 结果表明: 水体静止状态下沉积物一水界面溶解氧浓度随时间的增加而减 少, 非静止状态下随时间的增加而增加; 沉积物一水界面氧通量随水体流速的增加而增加.根据氧通量求解对应流速下垂 直浴动扩散系数并进行线性拟合, 当水体流速为 $0.01 \sim 0.14 \mathrm{~m} / \mathrm{s}$ 时, 垂直浴动扩散系数与水体流速的相关性最好, 此时 沉积物一水界面氧通量的传递以涡动扩散为主导.
\end{abstract}

关键词: 沉积物一水界面;浴度相关测试系统;水动力条件;氧通量;垂直浴动扩散系数;水体流速;御临河

\section{Influence of hydrodynamic conditions on the oxygen flux of sediment-water interface}

\author{
ZHENG Yanghua $^{1}$, ZOU Haodong ${ }^{2}$, HE Qiang ${ }^{2}$, LI Hong ${ }^{2}$, ZHANG Qing $^{3}$, CHEN Demin ${ }^{4} \&$ AI Hainan ${ }^{2 * *}$ \\ (1: Chongqing Environmental Protection Bureau, Chongqing 401147, P.R.China) \\ (2: Key Laboratory of the Three Gorges Reservoir Region's Eco-Environment, Ministry of Education, Chongqing University, \\ Chongqing 400044, P.R.China) \\ (3: Changsha Planning \& Design Institute Co., Ltd., Changsha 410000, P.R. China) \\ (4: Law School, Chongqing University, Chongqing 400044, P.R.China)
}

Abstract: Oxygen determines the final ending of various nutrient elements in sediments, and the sediment-water interface( SWI) is an important place for the oxygen transferring between the water phase and the sediments. This paper choose the Yulin River, one tributary of the Three Gorges Reservoir area, as the research object. According to the long-term monitoring data, a laboratory simulation device was established to simulate the natural water environment, and a non-invasive eddy correlation test system was built to explore the different hydrodynamic conditions on the SWI oxygen flux. The results showed that the dissolved oxygen concentration of SWI decreased with the increase of time in the stationary state of the water body, and increased with the increase of time in the nonstationary state. The SWI oxygen flux increases with the water velocity. According to the oxygen flux, the coefficient of vertical eddy diffusion $(K v)$ was calculated and linearly fitted. When the water velocity was $0.01-0.14 \mathrm{~m} / \mathrm{s}$, the correlation of the value of $K v$ and the water velocity was the best, and the SWI oxygen flux was transferred driven by eddy diffusion.

Keywords: Sediment-water interface; eddy correlation test system; hydrodynamic condition; oxygen flux; coefficient of vertical eddy diffusion; water velocity; Yulin River

沉积物是水体中物质与能量代谢的重要场所 ${ }^{[1]}$, 沉积物中氧环境决定了物质在沉积物中的赋存形态与 最终归趋 ${ }^{[2-7]}$. 沉积物中的氧主要来源于上覆水体中溶解氧的传递, 而沉积物一水界面 ( sediment-water inter-

* 国家自然科学基金项目 (51478061) 资助. 2018-01-06 收稿; 2018-03-02 收修改稿. 郑阳华(1982 ), 男,博士, 高 级工程师; E-mail:zhyh@ 126.com.

** 通信作者;E-mail:aihainan@126.com. 
face, SWI) 是氧传递发生的重要区域. 这种传递作用主要受以下几种条件的影响: (1) 沉积物的耗氧一一沉 积物中的耗氧过程主要有生物呼吸作用 (如底栖生物、微生物)、有机物质降解作用 (如有机碳化合物)、还 原性物质的氧化作用 (处于还原态的硫、铁、锰等); (2) 上覆水体中的氧浓度一一水体中氧浓度受大气复氧、 浮游植物光合作用产氧等影响; (3) 水体水动力条件一一氧从水相向沉积物相的传递方式包括分子扩散与 浴动扩散,水动力条件主要影响扩散的速率.

关于 SWI 氧通量的研究, 大部分集中在海洋 ${ }^{[8-10]}$, 近年来, 也有学者开始关注湖泊中氧的传递过程, 但 对于河流水体中氧传递过程却关注较少, 而且往往将 SWI 氧通量与水中营养盐等物质相关联 ${ }^{[11-15]}$. 然而, $\mathrm{SWI}$ 氧通量的大小除了与水体中的物质有关之外, 水动力条件也是影响它的重要因素. 目前, 关于水动力条 件对 SWI 氧通量影响的相关研究还比较少.

在准确表征 SWI 氧通量的产生、影响因素方面, 高精度的原位监测技术成为了主要制约因素. 随着计算 机科学的发展以及传感技术的进步,非侵人式浴度相关原位监测技术被逐步应用于 SWI 通量的研究中 ${ }^{[16]}$ 其理论基础是由 Montgomery (1948)、Swinbank (1951)、Obukhov (1951) 提出的 ${ }^{[17]}$, 核心在于对测试点流速与 氧浓度的同步监测. 该方法在过去的几十年内已经普遍应用于土壤一大气、海洋一大气、海洋一沉积物等界面 之间的 $\mathrm{CO}_{2}$ 、水汽等的通量测量 ${ }^{[18-19]}$, 近年来慢慢被应用于天然水体的通量原位监测 ${ }^{[20-21]}$. 如 McGinnis 等与 Brand 等 ${ }^{[22-23]}$ 分别采用浴度相关技术对 Wohlen 水库和 Alpnach 湖的 SWI 氧通量进行了研究, 结果表明浴度 相关技术是测量天然水体氧通量的一种极为有效的方法.

SWI 氧通量受外部环境条件影响较大, 特别是水动力条件. 因为水动力条件会改变氧在 SWI 的传质原 理, 而以往的研究对此关注较少. 论文选取三峡库区一级支流御临河为研究对象, 原位采集底泥沉积物与上 覆水, 模拟天然水体环境. 构建非侵人式浴度相关测试系统, 探究不同水动力条件对 SWI 氧通量的影响, 旨 在深化人们对水体 SWI 氧通量的理解.

\section{1 材料与方法}

\section{1 实验装置}

实验装置如图 1 所示. 该实验装置为长 $\times$ 宽 $\times$ 高 $=$ $1.2 \mathrm{~m} \times 1.0 \mathrm{~m} \times 0.8 \mathrm{~m}$ 的长方体水槽, 框体材质为有机 玻璃. 在距长方形水槽短边 $40 \mathrm{~cm}$ 处左右各设置一根 铁架,在铁架中心对称设置两台可调转速的搅拌转 子, 通过调节转速, 可模拟各种水动力条件. 装置正中 铺设有速度可调节的自动往返轨道, 多普勒测速仪可 固定在轨道下方自动往复. 轨道中间设置有可伸缩调 节高度的坚杆, 将溶解氧微电极固定于坚杆上, 调坚 杆节高度使微电极探头位于多普勒测速仪正下方. 实 验过程中所用多普勒测速仪与微电极测试系统通过 电缆各自连接多普勒测速仪主机及微电极测试系统 主机，用于将各自信号输出到计算机并通过相应软件

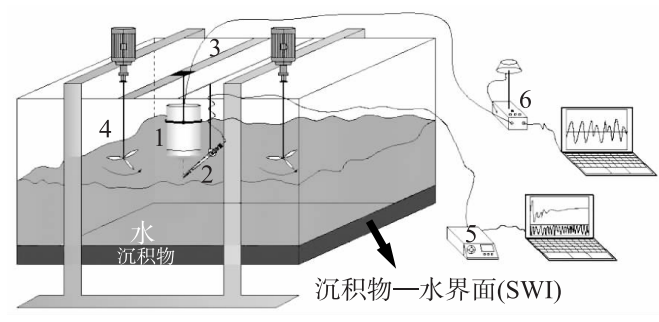

图 1 装置示意图

(1. 多普勒测速仪; 2 . 溶解氧微电极;

3. 自动往返轨道; 4 . 搅拌转子;

5. 微电极测试系统; 6 . 多普勒测速系统)

Fig.1 The schematic diagram of the device 转变为所需数据.

\section{2 实验材料}

研究采用的样品底泥沉积物及上覆水均采集于御临河 (图 2). 御临河 ( $29^{\circ} 34^{\prime} 45^{\prime \prime} \sim 30^{\circ} 07^{\prime} 22^{\prime \prime} \mathrm{N}, 106^{\circ} 27^{\prime}$ $30^{\prime \prime} \sim 106^{\circ} 57^{\prime} 58^{\prime \prime} \mathrm{E}$ ), 是三峡库区库尾、长江一级支流. 根据长年监测数据, 受三峡工程成库与回水影响, 御临 河在每年 4-9 月处于放水期, 平均流速约为 $0.05 \sim 0.33 \mathrm{~m} / \mathrm{s} ; 10$ 月一次年 3 月处于蓄水期, 平均流速约为 $0.01 \sim 0.08 \mathrm{~m} / \mathrm{s}$, 河流呈现明显的湖库型特征. 使用便携式沉积物采样器采集底泥, 所采底泥沉积物尽量减少 扰动, 保持原状并将其迅速放置于聚乙烯桶内密封保存, 运回实验室进行预处理. 将经预处理后的底泥均匀 平铺在装置底部, 厚度约为 $10 \mathrm{~cm}$. 上覆水用简易采水器于同一位置采集, 置于聚乙烯桶内, 运回实验室. 实 验开始前对上覆水进行测试, 高锰酸盐指数为 $12 \mathrm{mg} / \mathrm{L}$, 总氮浓度为 $1.8 \mathrm{mg} / \mathrm{L}$, 氨氮浓度为 $1.6 \mathrm{mg} / \mathrm{L}$, 总磷浓 


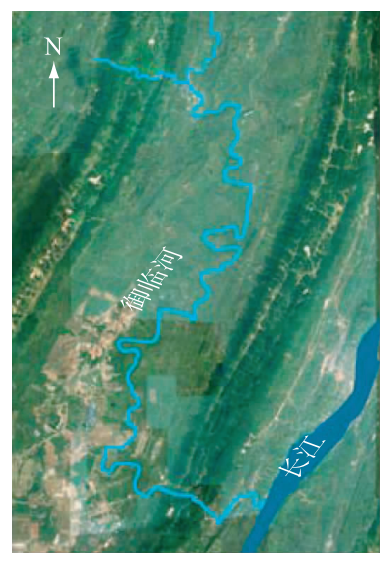

图 2 御临河区位

Fig. 2 The location of Yulin River

度为 $0.1 \mathrm{mg} / \mathrm{L}$. 用虹吸法将上覆水引人水槽, 控制水深为 $50 \mathrm{~cm}$, 实验室 温度 $25^{\circ} \mathrm{C}$, 气压 $981.45 \mathrm{hPa}$.

\section{3 非侵入式涡度相关测试技术}

浴度相关是一种基于湍流理论和统计分析的测试技术,采用非侵人 式浴度相关测试技术 ${ }^{[16]}$ 测量不同水动力条件下的 SWI 氧通量, 原理简 介如下.

水体中任意时刻和位置处, 控制水柱中由平流传输和分子扩散引起 的垂直氧通量 $O_{2} F l u x\left(\mathrm{mmol} /\left(\mathrm{m}^{2} \cdot \mathrm{s}\right)\right)$ 可表示为:

$$
O_{2} \text { Flux }=u_{z} \cdot C-D \frac{\mathrm{d} C}{\mathrm{~d} z}
$$

式中, $u_{z}$ 为垂直流速 $(\mathrm{m} / \mathrm{s}), C$ 为溶解氧浓度 $\left(\mathrm{mmol} / \mathrm{m}^{3}\right), D$ 为水中分子 扩散系数, $z$ 为垂直距离 $(\mathrm{m}), \frac{\mathrm{d} C}{\mathrm{~d} z}$ 为氧的浓度梯度 $\left(\mathrm{mmol} / \mathrm{m}^{4}\right)$.

因为湍流扩散在绝大部分自然水体扩散边界层中起主导作用, 分子 扩散作用可忽略,上式可简化为:

$$
\mathrm{O}_{2} \text { Flux }=u_{z} \cdot C
$$

此外, 由雷诺分解, 有 $u_{z}=\overline{u_{z}}+u_{z}^{\prime}$ (时均速度 + 脉动速度) 和 $C=\bar{C}+$

$C^{\prime}$ (时均浓度+脉动浓度)

假定 $\overline{u_{z}}=0$, 代人上式, 运用雷诺平均法则, 上式可进一步简化为:

$$
\overline{O_{2} \text { Flux }}=\overline{u_{z}^{\prime} \cdot C^{\prime}}=\frac{1}{N} \sum_{i=0}^{N-1} u_{z}^{\prime} \cdot C^{\prime}
$$

式中, $N$ 指实验中计算时段内获得的由溶解氧浓度及对应垂直流速组成的有效数据的组数, $\overline{O_{2} F l u x}$ 表示氧 通量在计算时段内的时均值. 该式表明, 某段时间内垂直方向上通过单位面积的氧通量大小等于水流的垂 直流速与氧脉动浓度的协方差.

实验原始数据由多普勒测速仪 (River Surveyor M9, 美国 SonTek 公司) 以及溶解氧微电极 ( OX25, 丹麦 Unisense 公司) 获得. 其中, 溶解氧微电极主要测量实验装置往返轨道中心点下方对应 SWI 中心点上方 (底 泥中心点上方 $10 \mathrm{~cm}$ ) 处的溶解氧浓度, 设置为每 $3 \mathrm{~s}$ 获取 1 个溶解氧数据. 多普勒测速仪主要测量不同水动 力条件下该点的垂直流速. 测得 SWI 中心点上方位置的垂直流速以及溶解氧浓度后, 利用上述浴度相关测 试技术即可计算出对应水动力条件下 SWI 的氧通量.

\section{4 垂直涡动扩散系数求解方法}

自然状态下的河流一般处于紊流状态. 水体的浴动程度可以用垂直浴动扩散系数 (Coefficient of vertical eddy diffusion, $K v)$ 来衡量. 垂直涡动扩散系数是对混合强度的一个度量, 用以表征在水体上、下层之间气体 和营养物质的交换, 以及动量和热量的交换 ${ }^{[24]}$. Imboden 和 Emerson ${ }^{[25]}$ 基于菲克(Fick) 方程,使用温跃层的 $K v$ 和缺氧湖下层与好氧混合层之间的磷浓度梯度计算出磷扩散进人混合层的通量. 类似地, 用溶解氧的 $K v$ 乘以水体上下层之间氧的垂直浓度梯度时, 得到的数值可以提供这两个区域之间单位面积氧的交换率, 即 氧通量. 计算公式如下:

$$
O_{2} \text { Flux }=K v \frac{\mathrm{d} C}{\mathrm{~d} z}
$$

式中, $K v$ 为垂直涡动扩散系数 $\left(\mathrm{m}^{2} / \mathrm{s}\right)$.

\section{5 实验设计}

根据长年监测结果, 选取 5 个不同的水动力条件, 通过依次调节搅拌转子转速, 使实验装置测量点在垂 直流速为 $0 \pm 0.01 、 0.03 \pm 0.02 、 0.07 \pm 0.02 、 0.12 \pm 0.02$ 和 $0.20 \pm 0.02 \mathrm{~m} / \mathrm{s}$ (平均流速为 $0 、 0.03 、 0.07 、 0.12$ 和 0.20 $\mathrm{m} / \mathrm{s}$ ) 的条件下分别运行 $1 \mathrm{~h}$, 即为 1 组实验. 每组实验重复进行 5 次. 


\section{2 结果与讨论}

\section{1 不同水动力条件下 SWI 氧通量的解析}

以平均流速为 $0 \mathrm{~m} / \mathrm{s}$ 为例, 展示利用浴度相关测试技术所获得的单组测试结果 (图 3). 当监测点垂直流 速介于 $-0.01 \sim 0.01 \mathrm{~m} / \mathrm{s}$ 之间时, 取 $3 \mathrm{~min}$ 为周期, 用微电极测得的瞬时溶解氧浓度 $C$ 计算该周期内溶解氧平 均浓度 $\bar{C}$, 之后由雷诺分解计算溶解氧脉动浓度 $C^{\prime}$; 用多普勒测速仪测得该点瞬时垂直流速 $u_{z}$, 假定 $\overline{u_{z}}=$ 0 , 则 $u_{z}^{\prime}=u_{z}$. 根据非侵人式涡度相关测试系统算法计算 $u_{z}^{\prime}$ 与 $C^{\prime}$ 的协方差, 即得该周期内 SWI 氧通量在 $-1.756 \sim 1.031 \mathrm{mmol} /\left(\mathrm{m}^{2} \cdot \mathrm{h}\right)$ 之间波动. 同理, 以 $30 \mathrm{~min}$ 为周期,计算得到静止状态下 $\mathrm{SWI}$ 氧通量为 -0.230 $\mathrm{mmol} /\left(\mathrm{m}^{2} \cdot \mathrm{h}\right)$, 负号表明, 氧在 SWI 由水相向沉积物相传递.

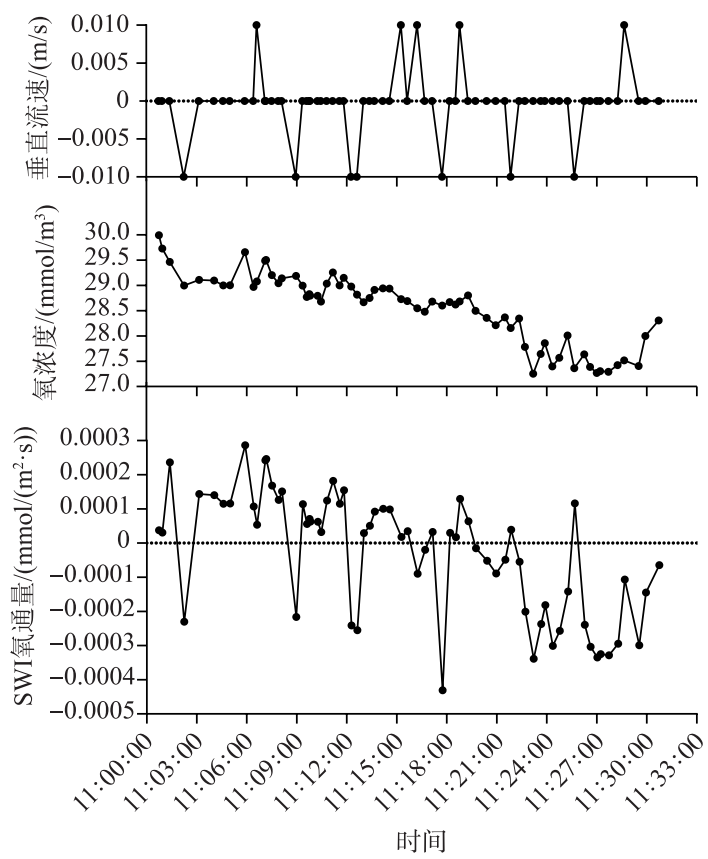

图 3 平均流速为 $0 \mathrm{~m} / \mathrm{s}$ 时监测点垂直流速、氧浓度和 SWI 氧通量

Fig.3 Vertical velocity, $\mathrm{O}_{2}$ concentration and SWI oxygen flux when the average velocity is $0 \mathrm{~m} / \mathrm{s}$

当平均流速为 $0 \mathrm{~m} / \mathrm{s}$ 时, 5 组实验中监测点的溶解氧浓度均随时间的增加而减少. 产生这种现象的原因 可能是水体在静止状态下, 氧从水相向沉积物相传递速度大于气相向水相复氧速度, 导致上覆水体中溶解 氧浓度不断降低, 在第 1 组实验中从 $29.994 \mathrm{mmol} / \mathrm{m}^{3}$ 降至 $27.424 \mathrm{mmol} / \mathrm{m}^{3}$, 溶解氧浓度变化量为 -2.570 $\mathrm{mmol} / \mathrm{m}^{3}$. 而当水体处于非静止状态, 即平均流速大于 $0 \mathrm{~m} / \mathrm{s}$ 时 (图 4), 监测点的溶解氧浓度均随时间的增 加而增加, 且变化幅度随扰动流速的增加而增加. 这可能是由于水体扰动使得大气复氧速率增加, 而氧在 SWI 中的传递除了受水动力条件的影响外, 还受其他因素的影响, 导致氧从水相传递至沉积物相出现滞后, 因而造成监测点溶解氧的累积.

依次提高搅拌速度改变水动力条件,重复进行 5 次实验,即为 1 组实验. 重复进行 5 组实验,利用上述计 算方法,得到氧通量(图 5).

在实验模拟的水动力条件范围内, SWI 氧通量大小随着平均流速的升高而升高, 在平均流速 $0 、 0.03$ 、 $0.07 、 0.12$ 和 $0.20 \mathrm{~m} / \mathrm{s}$ 下的氧通量平均值 \pm 标准差 $(\bar{x} \pm s)$ 分别为 $-0.213 \pm 0.022 、-0.561 \pm 0.080 、-0.952 \pm$ $0.092 、-1.561 \pm 0.140$ 和 $-2.955 \pm 0.094 \mathrm{mmol} /\left(\mathrm{m}^{2} \cdot \mathrm{h}\right)$, 误差在实验允许范围内.

\section{2 不同水动力条件对垂直涡动扩散系数的影响}

根据实验数据, 首先求出每 $3 \mathrm{~min}$ 周期测量点 $\mathrm{SWI}$ 氧通量, 然后利用公式 (4) 求解不同流速下 $K v$. 根据 

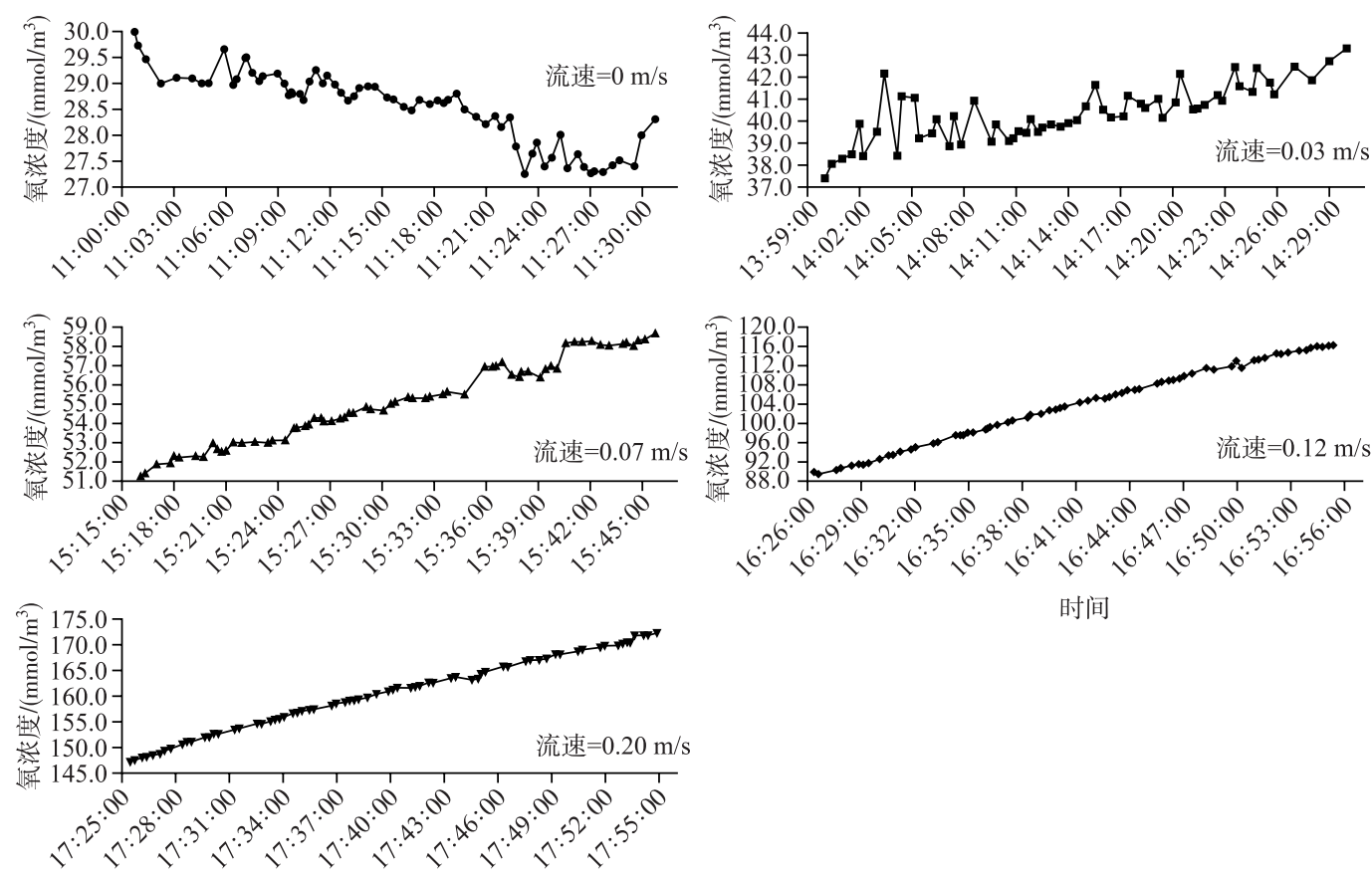

时间

图 4 监测点溶解氧浓度

Fig.4 The dissolved oxygen concentration of the monitoring point

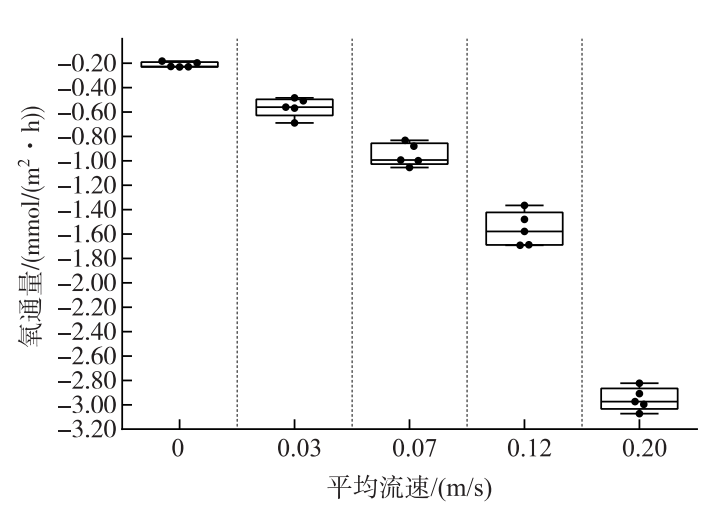

图 5 实验氧通量结果

Fig. 5 The oxygen flux results of the experiment

数据分布特点, 选择进行线性拟合, 建立水体流速 与 $K v$ 之间的单因素回归模型,得出不同水体流速 $u$ 与 $K v$ 的拟合函数 $K v=a u+b$. 其中 $\mathrm{a}$ 具有 $\mathrm{m}$ 的量 纲, $b$ 具有 $\mathrm{m}^{2} / \mathrm{s}$ 的量纲, 根据不同的流速区间, $\mathrm{a} 、 \mathrm{~b}$ 取值不同.

1 ) 水体流速为 $0 \sim 0.01 \mathrm{~m} / \mathrm{s}$ 时 SWI 的 $K v$ 模型 (图 6a). 当水体流速为 $0 \sim 0.01 \mathrm{~m} / \mathrm{s}$ 时, 溶解氧的 $K v$ 与水体流速的拟合函数为:

$$
K v=1.085 \times 10^{-7} u+8.662 \times 10^{-10}
$$

式中, $K v$ 为垂直浴动扩散系数 $\left(\mathrm{m}^{2} / \mathrm{s}\right) ; u$ 为水体流 速 $(\mathrm{m} / \mathrm{s})$.

此时, 垂直浴动扩散系数在 $0.9 \times 10^{-9} \sim 2.2 \times 10^{-9}$ $\mathrm{m}^{2} / \mathrm{s}$ 之间, 与 Hofman 等 ${ }^{[28]}$ 测定的氧在沉积物表层 的扩散系数 $\left(0.003 \times 10^{-9} \sim 0.010 \times 10^{-9} \mathrm{~m}^{2} / \mathrm{s}\right)$ 在数量级 上是一致的, 不同之处在于当水体流速接近 $0.01 \mathrm{~m} / \mathrm{s}$ 已不能与曲线较好的拟合. 另外, 垂直涡动扩散系数与 流速的 $R^{2}$ 值达到 0.8611 , 相关性较好. 结果表明, 当水体流速介于 $0 \sim 0.01 \mathrm{~m} / \mathrm{s}$ 之间时, 由于流速非常小, 此 时分子扩散与浴动扩散的共同作用造成 SWI 溶解氧的传递.

2 ) 水体流速为 $0.01 \sim 0.14 \mathrm{~m} / \mathrm{s}$ 时 SWI 的 $K v$ 模型 (图 $6 \mathrm{~b}$ ). 当水体流速为 $0.01 \sim 0.14 \mathrm{~m} / \mathrm{s}$ 时, 溶解氧的 $K v$ 与水体流速的拟合函数为:

$$
K v=1.165 \times 10^{-7} u+8.637 \times 10^{-8}
$$

此时, 水体处于紊动且底泥未悬浮状态, 溶解氧 $K v$ 随着水体流速的增加线性增加, 垂直浴动扩散系数 
与流速之间的 $R^{2}$ 值达到 0.9828 , 此阶段 SWI 氧通 量主要由垂直浴动扩散起主导作用, 数量级远远 大于分子扩散. 随着水体流速增大,引起 SWI 水体 的扰动, 扩散边界层 ( diffusive boundary layer, DBL) 厚度不断减少, 底边界层 (bottom boundary layer, BBL) 厚度不断增加, 紊动传质距离增加. 形 成较大的溶解氧扩散梯度, 沉积物作为汇, 溶解氧 由上覆水体源源不断向沉积物补充.

3 ) 水体流速为 $0.18 \sim 0.22 \mathrm{~m} / \mathrm{s}$ 时 SWI 的 $K v$ 模型 (图 6c). 当水体流速为 $0.18 \sim 0.22 \mathrm{~m} / \mathrm{s}$ 时, 溶 解氧的 $K v$ 与水体流速的拟合函数为:

$$
K v=2.686 \times 10^{-6} u-2.885 \times 10^{-7}
$$

此时底泥沉积物普遍开始悬浮, 水体开始浑 浊, 悬浮量随着速度的增加而增加. 沉积物中还原 性物质迅速耗氧,靠近底泥的 BBL 甚至可能形成 缺氧状态. 随着水体流速的增加, BBL 中涡动扩散 更加明显. 垂直浴动扩散系数与流速之间的 $R^{2}$ 值 仅为 0.1306. 其原因是沉积物再悬浮时,底泥中的 有机物和还原性物质与溶解氧的接触面积大大增 加并充分反应, 氧通量大幅增长, 而浴动扩散系数 不再仅受水体流速影响.

\section{3 不同水动力条件下 SWI 氧通量的变化规律}

由 5 组实验每小时氧通量变化量与变化率图 (此时氧通量变化量与变化率在数值上相等) 可 知,不同水动力条件下的氧通量变化量与变化率 呈现明显的三段式特征,具体表现为：

$0 \sim 1 \mathrm{~h}$, 水体处于静止状态, 平均流速为 $0 \mathrm{~m} / \mathrm{s}$, 此时有少量氧通量存在. $1 \sim 2 \mathrm{~h}$, 水体平均 流速为 $0.03 \mathrm{~m} / \mathrm{s}$, 水体由静止开始缓慢流动, 分子 扩散与浴动扩散共同起主导作用，上覆水体氧浓 度迅速增加, 变化率从无到有, 斜率较大, 平均为 $0.348 \mathrm{mmol} /\left(\mathrm{m}^{2} \cdot \mathrm{h}^{2}\right)$.

$2 \sim 3$ 和 $3 \sim 4 \mathrm{~h}$ 的水体平均流速分别为 0.07 和 $0.12 \mathrm{~m} / \mathrm{s}$, 此时水体处于紊动状态, 浴动扩散起主 导作用. 此时, 溶解氧由水相向沉积物相传递的大 小主要由水体流态决定, BBL 浴旋尺度在这个流
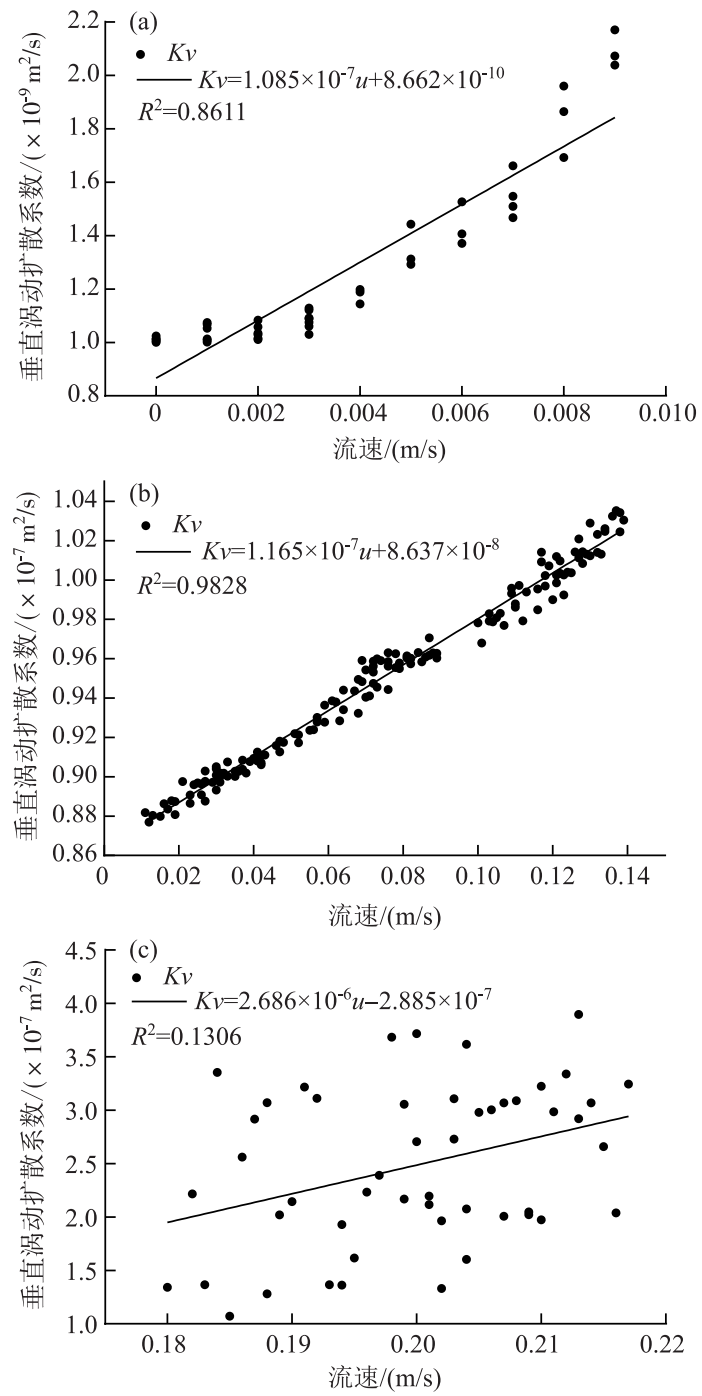

图 6 水体流速为 $0 \sim 0.01 \mathrm{~m} / \mathrm{s}(\mathrm{a}) 、 0.01 \sim 0.14 \mathrm{~m} / \mathrm{s}(\mathrm{b})$ 和 $0.18 \sim 0.22 \mathrm{~m} / \mathrm{s}(\mathrm{c})$ 时垂直浴动扩散系数随流速变化

Fig.6 The variety of vertical eddy diffusion coefficient with flow rate at the velocity of $0 \sim 0.01 \mathrm{~m} / \mathrm{s}(\mathrm{a})$, $0.01 \sim 0.14 \mathrm{~m} / \mathrm{s}(\mathrm{b})$ and $0.18 \sim 0.22 \mathrm{~m} / \mathrm{s}(\mathrm{c})$ 速区间增加较慢,变化率较为平缓, 氧通量变化率平均值分别为 0.390 和 $0.609 \mathrm{mmol} /\left(\mathrm{m}^{2} \cdot \mathrm{h}^{2}\right)($ 图 7$)$.

$4 \sim 5 \mathrm{~h}$, 水体平均流速变为 $0.20 \mathrm{~m} / \mathrm{s}$, 水体处于紊动状态. 同时, SWI 表层底泥开始悬浮,表层底泥与水 的接触面积大大增加, 沉积物中耗氧物质与氧充分反应, 微生物呼吸作用更为活跃, 氧浓度梯度大, 传质速 率高, 氧通量变化率达 $1.394 \mathrm{mmol} /\left(\mathrm{m}^{2} \cdot \mathrm{h}^{2}\right)$, 约为 $4 \mathrm{~h}$ 末的 2.3 倍. 此时, 浴动扩散系数不再仅受水体流速影 响(图 7).

\section{3 结论}

利用浴度相关技术测试了不同水动力条件下 SWI 氧通量大小, 分析了不同水动力条件下氧通量的变化 


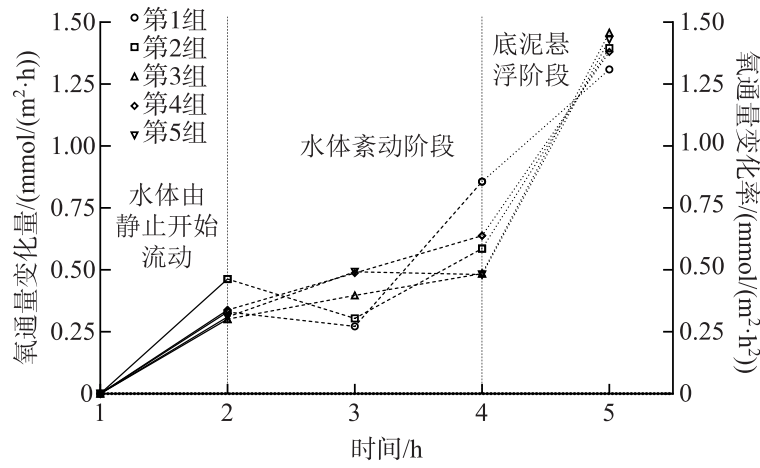

图 7 不同水动力条件下 SWI 氧通量的变化量与变化率

Fig.7 The change of SWI oxygen flux and the rate of change under different hydrodynamic conditions
规律和影响因素, 得出以下结论:

1) 不同水动力条件对 SWI 溶解氧浓度有 较大影响. 在静止状态下, 氧从水相向沉积物 相传递速度大于气相向水相复氧速度, 导致上 覆水体中溶解氧浓度不断降低; 在非静止状态 下, 由于水体存在扰动, 复氧速度与 SWI 氧传 递速度均随平均流速的增加不断增加, 而复氧 速度始终大于传递速度, 上覆水体溶解氧浓度 不断增加.

2)不同水动力条件对 SWI 氧通量的影响 显著, 随着平均流速的增加, 氧通量增加.

3 ) 不同水动力条件下, 将垂直涡动扩散系 数与平均流速进行线性拟合. 当水体流速为 $0 \sim 0.01 \mathrm{~m} / \mathrm{s}$ 时, $K v$ 与流速的相关性较好, $R^{2}=$ 0.8611 , 此时溶解氧受分子扩散和涡动扩散共 同影响; 当水体流速为 $0.01 \sim 0.14 \mathrm{~m} / \mathrm{s}$ 时, $K v$ 与流速的相关性最好, $R^{2}=0.9828$, 此时溶解氧以浴动扩散为 主; 当水体流速为 $0.18 \sim 0.22 \mathrm{~m} / \mathrm{s}$ 时, 溶解氧以浴动扩散为主, 但 $K v$ 与流速的相关性较差.

\section{4 参考文献}

[ 1 ] Wu FC, Wan GJ, Cai YR et al. Geochemical mechanisms controlling basic chemical compositions of lake water mass. Advances in Water Science, 1997, (2) : 73-79. [ 吴丰昌, 万国江, 蔡玉蓉等. 控制湖泊水体化学组成的地球化学原理 研究. 水科学进展, 1997, (2) : 73-79.]

[ 2 ] Rong N, Shan B. Total, chemical, and biological oxygen consumption of the sediments in the Ziya River watershed, China. Environmental Science and Pollution Research, 2016, 23(13): 13438-13447.

[ 3 ] Xiang SL, Tao SP, Wu DS et al. Exchange fluxes of phosphorus at the sediment-water interface in Lake Poyang. J Lake Sci, 2017, 29(2) : 326-333. DOI: 10.18307/2017.0208. [向速林, 陶术平, 吴代赦. 鄱阳湖沉积和水界面磷的交换 通量. 湖泊科学, 2017, 29(2): 326-333.]

[ 4 ] Hou D, He J, Lv C et al. Effects of environmental factors on nutrients release at sediment-water interface and assessment of trophic status for a typical shallow lake, northwest China. The Scientific World Journal, 2013, (3) : 716342.

[ 5 ] Wang JJ, Shen J, Zhang L et al. Sediment-water nutrient fluxes and the effects of oxygen in Lake Dianchi and Lake Fuxian, Yunnan Province. J Lake Sci, 2010, 22(5) : 640-648. DOI: 10.18307/2010.0503. [王建军, 沈吉, 张路等. 云南 滇池和抚仙湖沉积物-水界面营养盐通量及氧气对其的影响. 湖泊科学, 2010, 22(5): 640-648.]

[ 6 ] Atkinson CA, Jolley DF, Simpson SL. Effect of overlying water $\mathrm{pH}$, dissolved oxygen, salinity and sediment disturbances on metal release and sequestration from metal contaminated marine sediments. Chemosphere, 2007, 69(9) : 1428-1437.

[ 7 ] Zhu GW, Gao G, Qin BQ et al. Geochemical characteristics of phosphorus in sediments of a large shallow lake. Advances in Water Science, 2003, 14(6): 714-719. [ 朱广伟, 高光, 秦伯强等. 浅水湖泊沉积物中磷的地球化学特征. 水科学进 展, $2003, \mathbf{1 4}(6)$ : 714-719.]

[ 8 ] Ahmerkamp S, Winter C, Krämer K et al. Regulation of benthic oxygen fluxes in permeable sediments of the coastal ocean. Limnology and Oceanography, 2017, 62(5) : 1935-1954.

[ 9 ] Nakamura Y. Sediment oxygen consumption and vertical flux of organic matter in the Seto Inland Sea, Japan. Estuarine Coastal \& Shelf Science, 2003, 56(2): 213-220.

[10] Forster S, Graf G. Impact of irrigation on oxygen flux into the sediment: intermittent pumping by Callianassa subterranea and “piston-pumping” by Lanice conchilega. Marine Biology, 1995, 123(2) : 335-346.

[11] Fan CX, Morihiro A, Takehiko F et al. Study on the rate of the sediment oxygen demand in Kasumigaura Lake. Oceanologia et Limnologia Sinica , 1998, 29(5) : 508-513. [范成新, 相崎守弘, 福岛武彦等. 霞浦湖沉积物需氧速率的研究. 海洋与湖沼, 1998, 29(5) : 508-513.] 
[12] Zilius M, Daunys D, Petkuviene J et al. Sediment-water oxygen, ammonium and soluble reactive phosphorus fluxes in a turbid freshwater estuary (Curonian lagoon, Lithuania) : evidences of benthic microalgal activity. Journal of Limnology, $2012, \mathbf{7 1}(2): 33$.

[13] Xue LQ, Lu XW, Wu L et al. Dynamic simulation study on inner source nutrient exchange between water-sediment interfaces in the Xuanwu Lake. Advances in Water Science, 2004, (2): 189-192. [薛联青, 吕锡武, 吴否. 湖水-沉积物界 面内源物质交换动态复水模拟研究. 水科学进展, 2004, (2) : 189-192.]

[14] Grenz C, Denis L, Pringault O et al. Spatial and seasonal variability of sediment oxygen consumption and nutrient fluxes at the sediment water interface in a sub-tropical lagoon (New Caledonia). Marine Pollution Bulletin, 2010, 61 (7-12): 399-412.

[15] Bierlein KA, Rezvani M, Socolofsky SA et al. Increased sediment oxygen flux in lakes and reservoirs: the impact of hypolimnetic oxygenation. Water Resources Research, 2017, 53(6) : 4876-4890.

[16] Berg P, Roey H, Janssen F et al. Oxygen uptake by aquatic sediments measured with a novel non-invasive eddy correlation technique. Marine Ecology Progress, 2003, 261(8) : 75-83.

[17] Foken T. 50 years of the Monin-Obukhov similarity theory. Boundary-Layer Meteorology, 2006, 119(3) : 431-447.

[18] Wang B, Li J, Jiang WW et al. Impacts of the rangeland degradation on $\mathrm{CO}_{2}$ flux and the underlying mechanisms in the Three-River Source Region on the Qinghai-Tibetan Plateau. China Environmental Science, 2012, 32(10): 1764-1771. [王斌, 李洁, 姜微微等. 草地退化对三江源区高寒草甸生态系统 $\mathrm{CO}_{2}$ 通量的影响及其原因. 中国环境科学, 2012, 32(10) : 1764-1771.]

[19] Tao B, Ge QS, Li KR et al. Progress in the studies on carbon cycle in terrestrial ecosystem. Geographical Research, 2001, 20(5) : 564-575. [陶波, 葛全胜, 李克让等. 陆地生态系统碳循环研究进展. 地理研究, 2001, 20 (5): 564575. ]

[20] Shirasawa K, Ingram RG, Hudier JJ. Oceanic heat fluxes under thin sea ice in Saroma-ko Lagoon, Hokkaido, Japan. Journal of Marine Systems, 1997, 11(1/2) : 9-19.

[21] Xiao W, Liu SD, Li XH et al. Transfer coefficients of momentum, heat and water vapour in the atmospheric surface layer of a large shallow freshwater lake: A case study of Lake Taihu. J Lake Sci, 2012, 24(6) : 932-942. DOI: 10.18307/2012. 0617. [肖薇, 刘寿东, 李旭辉等. 大型浅水湖泊与大气之间的动量和水热交换系数一以太湖为例. 湖泊科学, 2012, 24(6) : 932-942.]

[22] Mcginnis DF, Berg P, Brand A et al. Measurements of eddy correlation oxygen fluxes in shallow freshwaters: Towards routine applications and analysis. Geophysical Research Letters, 2008, 350(4) : 222-256.

[23] Brand A, Mcginnis DF, Wehrli B et al. Intermittent oxygen flux from the interior into the bottom boundary of lakes as observed by eddy correlation. Limnology and Oceanography, 2008, 53(5) : 1997-2006.

[24] Kalff J ed. Limnology-Inland water ecosystem. Beijing: Higher Education Press, 2011: 188. [卡尔夫. 湖沼学一内陆 水生态系统. 北京: 高等教育出版社, 2011: 188.]

[25] Imboden DM, Emerson S. Natural radon and phosphorus as limnologic tracers: Horizontal and vertical eddy diffusion in Greifensee. Limnology and Oceanography, 1978, 23(1) : 77-90.

[26] Wang JN, Zhao L, Wei H. Variable diffusion boundary layer and diffusion flux at sediment-water interface in response to dynamic forcing over an intertidal mudflat. Chinese Science Bulletin, 2012, (8):656-665. [汪嘉宁, 赵亮, 魏皓. 潮滩 动力过程影响下扩散边界层和沉积物-水界面扩散通量的变化. 科学通报, 2012, (8) : 656-665.]

[27] Lorrai C, Mcginnis DF, Berg P et al. Application of oxygen eddy correlation in aquatic systems. Journal of Atmospheric \& Oceanic Technology, 2009, 27(9) : 1533-1546.

[28] Hofman P, Dejong SA, Wagenvoort EJ et al. Apparent sediment diffusion-coefficients for oxygen and oxygen-consumption rates measured with microelectrodes and bell jars-Applications to oxygen budgets in estuarine intertidal sediments (Oosterschelde, Sw Netherlands). Marine Ecology Progress Series, 1991, 69(3) : 261-272. 